\title{
Surface EBSD analysis and strengthening mechanism of AISI304 stainless steel subjected to massive LSP treatment with different pulse energies
}

\begin{abstract}
The effects of massive laser shock peening (LSP) treatment with different pulse energies on surface roughness and microstructural evolution in the surface layer of AISI304 stainless steel were investigated. The deformation-induced grain subdivision processes under two LSP treatment conditions of 3 and $6 \mathrm{~J}$ pulse energies were characterized and presented, respectively. Subsequently, EBSD characterization was conducted to analyze the peaks of misorientation angle for as-machined sample and LSPed samples with different pulse energies. Furthermore, a novel MT-MT intersection with four directions was found for the first time in the plastic deformation layer of AISI304 stainless steel, and the generation mechanism was completely presented according to the inherited crystal structure of austenite stainless steel. The formation process of the surface roughness and the formed microstructure subjected to massive LSP impact treatment with different pulse energies were compared and revealed.
\end{abstract}

Keywords: Laser shock peening; AISI 304 stainless steel; Plastic deformation; twin-twin intersection; Surface roughness; EBSD analysis 


\section{Introduction}

Austenitic stainless steels cannot be hardened by heat treatment because of low carbon content, and the amount of plastic strain induced strongly depends on the stress required to further deform the material [1,2]. Laser shock peening (LSP), which is also known as laser shock processing, is a surface strengthening technology that uses the mechanical effect of laser shock wave to generate a deeper compressive residual stress layer at the surface of metallic materials and alloys, and thus significantly improves the fatigue lives, wear, and corrosion resistance of metallic components $[3,4,5,6]$.

Over the past two decades, studies have reported the effects of LSP on the mechanical properties and microstructures of metal materials. For example, the effects of a single LSP impact on residual stress relaxation and hardness of the LSPed AISI 304 stainless steel (AISI 304SS) were investigated, and the results showed that LSP can improve the distributions of residual stress relaxation and hardness through the generated parallel mechanical twins (MTs) in one direction $[7,8]$. Similarly, the distribution of micro-hardness and micro-structural morphology for ANSI 321 stainless steel in depth direction were investigated before and after LSP, and the formation mechanism of the dislocation-cell structure in these stainless steels was established [9]. The increase in micro-hardness of the stainless steel subjected to LSP may also be attributed to increasing average dislocation density [10]. In fact, for metallic materials with low stacking fault energy, deformation twin dominates during plastic deformation $[11,12]$, and the MT thickness and the spacing between adjacent MTs increase with increasing strain rate [13]. Microstructure and fatigue life of dual-phase spring steel subjected to multiple laser peening have been investigated, and results showed that the ultra-high strain induced by laser shock wave was an important factor of grain refinement and plastic deformation in the surface layer of dual-phase spring steel [14]. Moreover, there was a transformation from interlath-retained austenites to martensites during warm laser peening [15]. 
The abovementioned studies concentrated on mechanical properties and deformation method by severe plastic deformation induced by laser shock wave. MT-MT intersection is the important form to refine the coarse grains of austenitic stainless steel suffering from evident surface treatment technologies, such as ultrasonic surface rolling [16], shot peening [17], and surface mechanical attrition treatment [18]. Considering quantitative research on multiple LSP impacts, we found MTs in the third direction for the first time in our previous work $[19,20]$ and reported the finding in the recent work [21]. We speculated whether MTs aligned in the fourth direction or the $n$th subdivided submicron triangular block into irregularly shaped submicron blocks with large misorientation would appear if the top surface of AISI 304SS was subjected to four or more LSP impacts [19]. Furthermore, the generation mechanism of MTs aligned in three directions is still pending. These proposed problems are worth investigating.

Tri-direction MT-MT intersection dominates during the grain refinement of AISI 304SS, which is induced by multiple LSP impacts. However, during LSP treatment, the relationship between MT direction and slip system in austenitic stainless steel was not found in previous reports. In engineering application, massive LSP treatment is an effective method to achieve uniform residual stress field, and different pulse energies result in different areas generating plastic deformation at the surface of metallic components. In addition, LSP treatment causes a coarser surface for austenitic stainless steel [8,22], and surface roughness meeting the requirement of engineering is another issue to consider.

This paper aims to investigate the effects of massive LSP treatment with different pulse energies on the surface roughness and grain refinement process of AISI 304SS. Special attention was given to the subdivision method by MT-MT intersection with multiple directions, and a relationship between MT direction and slip system was established. We revealed the formation of surface roughness and the formed microstructure subjected to massive LSP impact treatment with different pulse energies. 


\section{Experimental procedures}

\subsection{Sample preparation}

The AISI 304SS sample was cut into a rectangular plate with dimensions of $60 \mathrm{~mm} \times 20 \mathrm{~mm}$ with a thickness of $3 \mathrm{~mm}$. The chemical composition of AISI 304SS is listed in Table 1. Prior to LSP treatment, all samples were grinded using $\mathrm{SiC}$ paper with different grades of roughness (from 150 \# to 1500 \#), thoroughly degreased with ethanol, and then rinsed with deionized water by ultrasonic vibration.

\subsection{LSP experiment}

Massive LSP treatment was performed with a Q-switched Nd: YAG laser operated by a wavelength of $1064 \mathrm{~nm}$. The focused laser beam presents a diameter of $3 \mathrm{~mm}$ and pulse width of 10 ns. Two kinds of pulse energies of 3 and $6 \mathrm{~J}$ were selected. During massive LSP treatment, a layer of flowing water with 1-2 mm thick was used as confinement layer to enhance the peak pressure induced by laser shock wave, and the $3 \mathrm{M}$ professional aluminum foil (made in USA) with a thickness of $100 \mu \mathrm{m}$ was selected as the absorbing layer to prevent the thermal effect on the sample surface.

The peak power density can be calculated according to the following formulas:

$$
\begin{aligned}
& Q_{P}=\frac{E_{P}}{(A \tau)} \\
& \mathrm{A}=\frac{\pi d^{2}}{4}
\end{aligned}
$$

where $A$ is the beam shot area $\left(7.07 \mathrm{~mm}^{2}\right), \tau$ is the laser pulse duration (10 ns), and $E_{P}$ is the pulse energy. Hence, the peak power densities are equal to 4.24 and $8.48 \mathrm{GW} / \mathrm{cm}^{2}$ when the pulse energies are 3 and $6 \mathrm{~J}$, respectively.

As shown in Fig. 1, Region A (in gray) and Region B (in light blue) on the AISI 304SS sample surface were treated using massive LSP treatment with pulse energies of 3 and $6 \mathrm{~J}$, respectively. The 
red point denotes the starting point, and the blue point denotes the end point on the top surface. The scanning path and overlapping rate of massive LSP treatment can be found in the literature [23]. After massive LSP treatment, the treated sample was uniformly cut into three thin plates in depth direction, and the bottom part is regarded as substrate (as-received sample) S1. Subsequently, the top part is divided into two pieces, i.e., the LSPed region with a pulse energy of $3 \mathrm{~J}$ (S2) is on the left, and the LSPed region with a pulse energy of $6 \mathrm{~J}(\mathrm{~S} 3)$ on the right. Three kinds of samples are schematically illustrated in detail in Fig. 1.

\subsection{Surface roughness measurement and microstructural characterization}

Surface roughness data of all three kinds of samples (S1, S2, and S3) were determined using a true color confocal microscope (Axio CSM 700), and the average value was adopted. The detailed measurement regions were schematically described as A, B1, and B2, as presented in Fig. 1. Typical microstructures of all three kinds of samples ( $\mathrm{S} 1, \mathrm{~S} 2$, and $\mathrm{S} 3$ ) were observed via transmission electron microscopy (TEM, JEM-2100). For the LSPed samples (S2 and S3), the TEM foils taken from the top surface and a depth of $30 \mu \mathrm{m}$ below the top surface were prepared. Hence, four TEM foils, namely, B1, B2, C1, and C2, can be schematically presented, as shown in Fig. 1. The electron backscattered diffraction (EBSD) analysis was performed to investigate the misorientation and texture. Before EBSD test, all samples were ground with SiC papers (from 150 \# to 1500 \#), mechanically polished with diamond paste with $\mathrm{W} 1.0$ and $\mathrm{W} 0.5$ and then were finely electrochemical polished for one hour. Next, EBSD was performed on a FEI Nano SEM Nova 430 equipped with an Oxford Instruments Nordlys $2 \mathrm{~S}$ detector. The step size is $0.5 \mu \mathrm{m}$. The HKL

Channel 5 software was used to analyze and display the data. In the present work, the EBSD analyses were conducted in Regions A, B1, and B2. 


\section{Results and discussions}

\subsection{Surface topography and roughness}

Fig. 2 shows the microstructural topographies of the as-received sample and LSPed samples, including 2D and 3D microstructural morphologies. Fig. $2 \mathrm{a}$ and Fig. $2 \mathrm{~b}$ present the three-dimensional profile of the LSPed samples (S2 and S3) within a measurement region of $579 \mu \mathrm{m}$ $\times 400 \mu \mathrm{m}$ of the top surface, respectively. Abundant shallow and narrow micro-grooves were observed in the top surface of the sample S2, which may inherit fractional surface feature of the as-received sample [24]. After massive LSP treatment with a pulse of $6 \mathrm{~J}$, apart from micro-grooves, several conspicuous scattered micro-bugles can be found. Figs. $2 \mathrm{c}$ to $2 \mathrm{e}$ are the two-dimensional surface profiles of three kinds of samples (S1, S2, and S3), respectively. The surface profiles of S2 and S3 with a length of $200 \mu \mathrm{m}$ are taken from two lines (L1 and L2) perpendicular to the scratch direction, respectively. The surface profile of $\mathrm{S} 1$ fluctuates between -3.5 and $3.7 \mu \mathrm{m}$, whereas that of $\mathrm{S} 2$ fluctuates between -4 and $5 \mu \mathrm{m}$. As the pulse energy increases to $6 \mathrm{~J}$, the surface profile varies between -5 and $3.5 \mu \mathrm{m}$.

Table 2 presents the average value (AVG) of surface roughness for the as-received sample and LSPed samples within a measurement length $(l r)$ of $0.80 \mathrm{~mm}$. As seen from Table 2, the surface roughness of the as-received (S1) is $0.536 \mu \mathrm{m}$, which is obviously lower than 1.226 (S2) and 0.947 $\mu \mathrm{m}$ (S3) of both LSPed samples. These data are in a good agreement with the measurement values in surface profiles, indicating that massive LSP treatment increases the surface roughness of the as-received sample. The phenomenon is due to the increase in surface waviness [24]. Notably, the surface roughness of S3 is lower than that of S2 $(0.947 \mu \mathrm{m}$ versus $1.226 \mu \mathrm{m})$, indicating that higher pulse energy can cause a more uniform surface roughness.

\subsection{Micro-structural evolution after LSP with different pulse energies}

Our previous work [19] showed that there was a serve plastic deformation (SPD) layer with a depth of $20 \mu \mathrm{m}$ and the following minor plastic deformation (MPD) layer along the depth direction 
of AISI 304SS samples subjected to two LSP impacts. Therefore, in the present work, the TEM images were taken from the top surface to a depth of $30 \mu \mathrm{m}$ below the top surface of both LSPed samples.

Figs. 3a to 3c show typical TEM images on the top surface of the LSPed sample with a pulse energy of $3 \mathrm{~J}$. As seen from Fig. 3a, three coarse grains are subdivided by triple-direction MTs or MTs with two directions (R2). There are a number of MTs with three directions in coarse grains R1 $\underline{\text { and R3 }}$, whereas coarse grain R2 is divided by MT-MT intersection into rhombic blocks. Fig. 3b is a magnified image of the rectangle $[\mathrm{M}]$ in Fig. 3a. Fig. 3b shows several MT-MT intersections with three directions, namely, D1, D2, and D3, as shown by the yellow dashed arrows in the right upper corner. The width of MT aligned in D1 direction, $\lambda_{1}{ }^{\mathrm{D} 1}\left(\lambda_{1}{ }^{\mathrm{Dn}}\right.$ represents the width of MT aligned in Dn direction) is calculated as $20 \pm 5 \mathrm{~nm}$. The spacing of neighboring MTs along D1 direction, $\underline{s}_{1} \underline{\text { D1 }}$ $\left(s_{1}{ }_{1}^{\text {Dn }}\right.$ denotes the spacing of neighboring MTs along Dn direction $)$ is $180 \pm 20 \mathrm{~nm}$. Both $\lambda_{1}{ }^{\mathrm{D} 2}$ and $s_{1}{ }^{\mathrm{D} 2}$ are $90 \pm 10 \mathrm{~nm}$. Both $\lambda_{1}{ }^{\mathrm{D} 3}$ and $s_{1}{ }^{\mathrm{D} 3}$ are $140 \pm 20$ and $500 \pm 50 \mathrm{~nm}$, respectively. Fig. $3 \mathrm{c}$ is the corresponding selected-area electron diffraction (SAED) pattern taken from the ellipse [A] in Fig. 3b. The corresponding diffraction spots display mirror symmetry with respect to a common $\{111\}$ plane, which is approximately parallel to the twin plane (Fig. 4c). This finding indicates that the twin segment $\mathrm{T}$ represented a fine $\{111\}<112>$ twin, which was further confirmed by the misorientation angle/axis pair of $\sim 60^{\circ}<111>$ in the subsequent EBSD analysis.

Figs. $3 \mathrm{~d}$ to $3 \mathrm{f}$ are the typical TEM images obtained from a depth of $30 \mu \mathrm{m}$ below the top surface of the LSPed sample with a pulse energy of $3 \mathrm{~J}$. As seen from Fig. 3d, the coarse grain exceeds $7 \mu \mathrm{m}$ and a large number of multiple-directional MTs are presented in every grain. Fig. 3e is the magnified image of the rectangle $[\mathrm{N}]$ in Fig. $3 \mathrm{~d} . \lambda_{2}{ }^{\mathrm{Dn}}$ and $\mathrm{s}_{2}{ }^{\mathrm{Dn}}$ represent the MT width and the spacing between two adjacent MTs in the $n$th direction, as shown in Figs. $3 e$ and $3 \mathrm{f}$, respectively. In the coarse grain, a large number of MTs in three directions (D1, D2, and D3) are generated by laser shock wave. $\lambda_{2}{ }^{\mathrm{D} 1}$ varies from $20 \mathrm{~nm}$ to $70 \mathrm{~nm}$, whereas $s_{2}{ }^{\mathrm{D} 1}$ is approximately $130 \mathrm{~nm}$. At the bottom 
of this image, $s_{2}{ }^{\mathrm{D} 1}$ reaches $250 \mathrm{~nm} . \lambda_{2}{ }^{\mathrm{D} 2}$ and $s_{2}{ }^{\mathrm{D} 2}$ are $20 \pm 5$ and $280 \pm 50 \mathrm{~nm}$, respectively. Along the

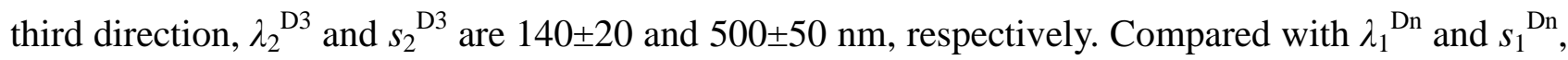
the MTs along D2 direction become sparser and narrower with increasing depth away from the top surface. Most of MTs along D1 direction also become sparser and narrower. However, MTs along the D3 direction with a larger thickness of $200 \mathrm{~nm}$ exist in the coarse grain, and the spacing between two adjacent MTs varies from $500 \mathrm{~nm}$ to several micrometers. In addition, the twins of D3 are virtually unchanged. Fig. 3f is the corresponding dark-field TEM image of Fig. 3e, in which the high-resolution MTs can be clearly observed. As seen from the enlarged image in the square B shown in the inset of Fig. 3f, martensite transformation may be induced by massive LSP treatment, as is in accordance with the previous result [21]. This phenomenon can be explained as follows: when the sample surface is frequently impacted during massive LSP treatment, a part of mechanical energy within grains is transferred into heat energy because of collision among atoms; this collision causes the occurrence of martensite phase transformation.

Figs. 4a to 4c show the typical TEM images on the top surface of the LSPed sample (S3) with a pulse energy of $6 \mathrm{~J}$. As found in Fig. 4a, the coarse grain is approximately $5 \mu \mathrm{m}$. At the lower right corner, multiple-directional MT-MT intersections can clearly be observed, as shown in the ellipse [A]. The appearance of the MT in the fourth direction, which is a novel phenomenon, is particularly notable. Fig. $4 \mathrm{~b}$ is the magnified image of the rectangle $[\mathrm{M}]$ in Fig. 4a. A typical tri-directional MT-MT intersection can be observed. Their MT widths along D1, D2, and D3 directions are 85 \pm 20 , $150 \pm 20$, and $40 \pm 5 \mathrm{~nm}$, and their corresponding spacings are $100 \pm 25,350 \pm 50$, and $360 \mathrm{~nm}$, respectively. For characterizing the microstructure of MTs, the SAED pattern taken from the ellipse B in Fig. 4b is shown in Fig. 4c. This pattern, which includes two adjacent bands, is taken with the [110] zone axis, and the mirror spots of the [110] diffraction pattern appear with respect to the $\{\overline{1} 11\}$ plane. These findings indicate that the two adjacent bands constitute the twin structure.

Figs. $4 \mathrm{~d}$ to $4 \mathrm{f}$ are the typical TEM images at a depth of $30 \mu \mathrm{m}$ below the top surface of the Page-8- 
LSPed sample with a pulse of $6 \mathrm{~J}$. The coarse grain is larger than $6 \mu \mathrm{m}$. Similar to the microstructure on the top surface, MT-MT intersection with four directions can be found in Fig. 4e. Fig. $4 \mathrm{f}$ is the magnified image of the rectangle $[\mathrm{N}]$ in Fig. 4e, showing MTs along D1, D2, D3, and D4 directions. The MT widths along four directions are 25 $\pm 5,125 \pm 20,50 \pm 5$, and $100 \pm 20 \mathrm{~nm}$, and their corresponding spacings are $500 \pm 50,625 \pm 50,580 \pm 50$, and $1050 \pm 50 \mathrm{~nm}$, respectively. Hence, with increasing distance from the top surface, the MT width decreases, whereas the spacing between two adjacent MTs gradually increases. In addition, with increasing pulse energy, the MT width increases, but the spacing remains unchanged.

\subsection{EBSD analyses of three kinds of samples}

Fig. 5 presents the EBSD maps of three kinds of samples (S1, S2, and S3). We employed [100] solely as the reference direction for the inverse pole figure to code the color pattern, as indicated by the triangle in top right corner of Figs. 5a, 5c, and 5e. The histograms of number percentage versus misorientation angle between neighboring grains are also shown in the second row.

Fig. 5a displays the initial microstructure, which consists of equiaxed austenitic grains, of the as-received sample $\mathrm{S} 1$. The average size of the coarse grain exceeds $30 \mu \mathrm{m}$. The grain boundary misorientation distributions of $\mathrm{S} 1$ can be seen. The misorientation angle is defined as the minimum rotation angle required for two neighboring crystal lattices to coincide. Moreover, the number percentage at a certain misorientation angle is defined as the total number of pixels along all grain boundaries within the analysis area divided by the number of pixels along the boundaries within this misorientation angle. Given that a grain boundary in an EBSD can be distinguished by pixels with misorientation angles beyond the specified gain tolerance angle, such as $2^{\circ}$, in the present analysis, the number percentage within a given range actually denotes the fraction of the boundary length relative to the total length of all grain boundaries. Only $5 \%$ of grain boundaries were found with small misorientation angles less than $15^{\circ}$. In particular, $2^{\circ}$ misorientation angles of grain boundaries are minimal. Meanwhile, more than $80 \%$ of boundaries have large misorientation angles ranging 
from $20^{\circ}$ to $55^{\circ}$, as shown in Fig. 6 b. The experimental histogram of as-received sample follows the random distribution fairly well.

For the LSPed sample with a pulse energy of $3 \mathrm{~J}(\mathrm{~S} 2)$, abundant grains with different sizes were observed, as depicted on the right side of Fig. 5c. The grain size in the ellipse [A] is approximately $40 \mu \mathrm{m}$, which is equal to the size of the original grain. After massive LSP treatment with a pulse energy of $3 \mathrm{~J}$, the grain on the top surface decreases in size (to only $5 \mu \mathrm{m}$ ), as shown in the ellipse [B]. The distribution of misorientation angles shown in Fig. 5d reveals the relationship between both adjacent grains. As seen from the histogram of number percentage versus misorientation angle taken from the surface of S2, approximately $7 \%$ of grain boundaries possess small misorientation angles of less than $15^{\circ}$. A sharp contrast with those of the as-received sample is that the quotient, which comprises approximately $3 \%$ of grain boundaries, presents a misorientation angle near $2^{\circ}$. Another approximately $46 \%$ of grain boundaries possess large misorientation angles ranging from $20^{\circ}$ to $55^{\circ}$. A high percentage of MTs appears as a sharp peak at $60^{\circ}$ on the misorientation angle distribution. The MTs in face-centered cubic (FCC) metals are $\sum 3$ coincidence site lattice boundaries, which are rotated by $60^{\circ}$ around the densely packed direction [111].

For the LSPed sample with a pulse energy of $6 \mathrm{~J}(\mathrm{~S} 3)$, homogeneous, fine, and equiaxed grains with a mean grain size of $15 \mu \mathrm{m}$ are achieved as shown in Fig. 5e. Compared with S2, more uniform grains are presented on the top surface of S3. As far as the relation of boundary number percentage versus misorientation angle is concerned, the boundaries of misorientation angle from $20^{\circ}$ to $55^{\circ}$ also account for $49 \%$, whereas the percentage of misorientation angle from $40^{\circ}$ to $50^{\circ}$ increases compared with those of S2 and S1. Meanwhile, the peak of $60^{\circ}$ shares a large contribution of $40 \%$, which is less than that on S2. In addition, another marked difference between S3 and S2 lies in the number percentage of small misorientation angles of $2^{\circ}$. The number percentage of $\mathrm{S} 3$ decreases to 0 compared with $3 \%$ of $\mathrm{S} 2$, which is similar to that of the as-received sample, as shown in Fig. $5 \mathrm{~b}$. These variations reveal that the recrystallization has been accomplished. The peak of $60^{\circ}$ 
misorientation angle in Fig. 5a reaches 9\%, which is different from 46\% (S2) and 40\% (S3), as shown in Figs. $5 \mathrm{~d}$ and $5 \mathrm{f}$ in nature because grains with $60^{\circ}$ misorientation do not always possess a twin relationship. When the samples were subjected to LSP (Fig. 5d and f), a peak of increasing intensity appears at $60^{\circ}$, corresponding to twin boundaries. This phenomenon can be explained by the following fact. The EBSD maps of $\mathrm{Au}-\mathrm{Cu}-\mathrm{Ag}$ alloys without and with 20 and $200 \mathrm{ppm}$ Ir additions were characterizd, and results showed that when an increasing amount of Ir was added, a peak of increasing intensity appears at $60^{\circ}$ which corresponds to twin boundaries, since a twin corresponds to a rotation of $60^{\circ}$ of the lattice around a common <111> direction [25] After LSP, a number of deformation twins of $\sim 60^{\circ}<111>$ are presented in the surface layer of AISI 304SS, as is in accordance with the experimental results in Section 3.2. As seen from Fig. 5, the coarse grains are refined by LSP, which is consistent with the results of our previous research [19].

\subsection{Schematic representation of grain refinement induced by LSP impact with different energy}

Fig. 6 schematically illustrates the formation of the surface roughness and microstructure subjected to massive LSP treatment with different pulse energies. As seen in Section 3.1, massive LSP treatment causes an increase in surface roughness. However, surface roughness of S3 subjected $\underline{\text { to LSP }}$ with a pulse energy of $6 \mathrm{~J}$ is evidently lower than that with a pulse energy of $3 \mathrm{~J}$ ( 0.947 versus 1.226). In materials affected by shock waves, when the peak pressure resulting from the shock wave exceeds the Hugoniot elastic limit (HEL) of the metallic materials and alloys, plastic deformation will occur in the surface layer [26]. Subsequently, micro-dimples will be generated in the surface layer, thereby resulting in higher surface roughness compared with that of the as-received sample [27].

In the present work, the laser pulse follows the spatial distribution of a similar Gauss. Owing to the spatial non-uniformity of pulse energy, the effective deformation area will decrease when the peak pressure of laser shock wave is not sufficiently high. Hence, for different pulse energies of 3 and $6 \mathrm{~J}$, their effective deformation areas differ, with the area of $6 \mathrm{~J}$ being evidently larger than that 
of $3 \mathrm{~J}$.

As illustrated in Fig. 6a, when AISI 304SS is treated by massive LSP treatment with $3 \mathrm{~J}$ pulse energy, a micro-indention with a height of $\mathrm{H} 1$ forms. After a successive LSP impact with 50\% overlapping rate, the height of the first micro-indention decreases to H2. Hence, after massive LSP treatment, the difference between the peak and the valley is $\mathrm{H} 2$. Notably, the diameter of the effective deformation area is L1, which is less than the diameter of the laser spot. Similarly, when AISI 304SS is treated by massive LSP treatment with $6 \mathrm{~J}$ pulse energy, a micro-indention with a larger height of $\mathrm{H} 3$ also appears on the top surface. After the second LSP impact with 50\% overlapping rate, the height of the first micro-indention decreases to $\mathrm{H} 4$.

Compared with the diameter L1 of the effective deformation area with $3 \mathrm{~J}$ pulse energy, L2 with $6 \mathrm{~J}$ pulse energy is relative large, as shown in Fig. 6. Thus, during massive LSP treatment, most of the overlapping region is covered by one LSP impact (in white) or two LSP impacts (in blue) when the pulse energy is $3 \mathrm{~J}$. For S3, the majority of the overlapped region is covered by three LSP impacts (in yellow) or four LSP impacts (in red), as shown in both front views of the two cases. In fact, multiple LSP impacts can generate a smoother surface of metallic materials and alloys [28]. Hence, massive LSP treatment with $6 \mathrm{~J}$ pulse energy can result in a smoother surface than $3 \mathrm{~J}$ pulse energy on the top surface of AISI 304SS.

In addition, MT-MT intersections with four directions are observed in the surface layer subjected to massive LSP treatment with $6 \mathrm{~J}$ pulse energy. The appearance of the MT in the fourth direction is a novel phenomenon, which was observed for the first time. This phenomenon may be explained as follows. According to our previous work [19], three LSP impacts generate MTs with three directions in the coarse grain of AISI 304SS. In the present work, a part of the overlapped region is impacted for four times, leading to the occurrence of MTs in the fourth direction. For S2, the grain refinement mechanism has been presented in the literature [19], and MTs with four directions are almost not found in the surface layer. 
In contrast, as seen from Fig. 6b, the area (in red) covered by four LSP impacts accounts for the larger proportion. The TEM images in Fig. 4 shows the intersections of MT-MT in four directions after four LSP impacts. Mechanical twins are major deformation mechanism caused by applied $\underline{\text { stress and deformation mechanism dominated by MT is highly confined on the }\{111\} \text { slip plane in }}$ austenitic steels. The formula [29] proposed by Byun shows the relationship of critical stress for twin in terms of the equivalent or uniaxial stress and $\gamma_{S F}$ :

$$
\sigma_{T}=6.14 \frac{\gamma_{S F}}{b_{P}}
$$

In accordance with this formula, the critical stress is $\sim 700 \mathrm{MPa}$ (here, $\gamma_{\mathrm{SF}}=14 \mathrm{~mJ} / \mathrm{m}^{2}, b_{\mathrm{P}}=$ $0.147 \mathrm{~mm})$. In the present work, the corresponding peak pressures of $3 \mathrm{~J}$ and $6 \mathrm{~J}$ are $3.83 \mathrm{MPa}$ and 5.42 $\mathrm{MPa}$, respectively, which are much higher than the critical stress. Hence, the generated laser shock wave can cause the twin deformation, as shown in TEM observations (Figs. 3 and 4).

After four LSP impacts, MTs in four directions nucleate, grow, and eventually transform into equiaxed refined grains through the development of the subgrain boundaries. Subsequently, continuous dynamic recrystallization may occur [19,29]. Finally, the refined grains form in the surface layers of AISI 304SS with further increase in the strain induced by laser shock wave (shown in Fig. 7c), as illustrated in Fig. 5e.

The twin planes in FCC alloys are invariably the close-packed $\{111\}$ planes, whereas the twin directions are [112]. Fig. 8a is a three-dimensional representation of the four $\{111\}$ twin planes in a FCC crystal [30], and Fig. 8b shows a computing method of the interplanar angles of $\{111\}$ crystal family planes. The interplanar angles of $\{111\}$ twin planes are all equal to $70.5^{\circ}$, and $109.5^{\circ}$ is the supplementary angle of $70.5^{\circ}$. Fig. 8c shows a lattice with a three-layer twin after shear displacement $\boldsymbol{u}$ (indicated by the black arrow) in successive (111) planes. The twin plane is marked by the red dashed line. TEM observations are performed with the grain orientation adjusted to a [110] 
orientation. In this orientation, (111) and (111) twins are inclined to the surface, whereas (111) and (111) twins stand sideways to the surface along [110] beam direction, as shown in Fig. 8a. AISI 304SS possesses a FCC crystal structure with four twin systems with twin planes $\{111\}$ and relevant twin directions [112]]; $\{111\}$ corresponds to the family of twin planes $\boldsymbol{n}$ and [112] corresponds to the family of twin directions $s$, respectively. The detailed twin system of AISI 304SS is shown in Table 3.

In our previous study [19], MTs aligned in three directions subdivided the coarse grains into submicron triangular blocks at the top surface of the AISI 304SS after three LSP impacts. Given that FCC alloys with a low SFE, such as AISI 304SS, 316L stainless steel, and IN 718, possess four twin planes. These alloys will generate twins aligned in four directions under the most severe plastic deformation with an ultra-high strain rate. Hence, we can conclude the existence of MTs with four directions in the plastic deformation layer of the FCC alloys with a low SFE. These MTs strongly depend on the impact time at micro-region and the inherited crystal structure of the FCC alloys with a low SFE.

MT dominates during the plastic deformation of AISI 304SS induced by laser shock wave (Fig. 7). In Section 3.2, $\varepsilon$-martensite is also observed at a depth of $30 \mu \mathrm{m}$ after massive LSP treatment with $3 \mathrm{~J}$ pulse energy, which is similar to phase transformation from austenite grains to $\alpha^{\prime}$-martensite of AISI 316L stainless steel caused by severe shot peening [2], confirming grain refinement. For the stacking fault energy (SFE), namely, $\gamma_{\mathrm{SF}}<18 \mathrm{~mJ} / \mathrm{mol}$, the $\varepsilon$-martensitic transformations occurs, whereas mechanical twin takes place for $12 \mathrm{~mJ} / \mathrm{mol}<\gamma_{\mathrm{SF}}<35 \mathrm{~mJ} / \mathrm{mol}[31,32]$.

Based on the microstructural observation and our previous results, the grain refinement of AISI 304SS subjected to massive LSP treatment with $6 \mathrm{~J}$ pulse energy can be schematically illustrated in the following three steps: (i) high-density micro-MTs divide the coarse grains into micrometer-thick T-M lamellae; (ii) MTs with four directions further subdivide the T-M lamellae into equiaxed 
micro-sized and irregularly shaped blocks; and (iii) the subdivided blocks evolve into randomly refined grains. According to the empirical formula proposed by Schramm and Reed, the SFE of AISI $304 \mathrm{SS}$ is $\sim 14 \mathrm{~mJ} / \mathrm{m}^{-2}$ [33]. As a consequence, MT also occurs during the plastic deformation induced by laser shock wave, which agrees well with the literature [21]. Hence, MT-MT intersection is the major deformation mechanism of AISI 304SS subjected to massive LSP impacts.

\section{Conclusions}

The effects of massive LSP treatment with different pulse energies on surface roughness, microstructural evolution, and grain refinement mechanism of AISI304 stainless steel are investigated, and EBSD analyses of three kinds of samples are also performed. Several important conclusions can be made as follows:

(1) Massive LSP treatment causes an increase in surface roughness of the as-received sample, but massive LSP treatment with $6 \mathrm{~J}$ pulse energy results in a relative smooth surface with a surface roughness of $0.947 \mu \mathrm{m}$ compared with that of $1.226 \mu \mathrm{m}$ with $3 \mathrm{~J}$ pulse energy. This phenomenon can be attributed to the fact that the $6 \mathrm{~J}$ pulse energy presents a relatively large effective deformation area compared with the $3 \mathrm{~J}$ pulse energy.

(2) Compared with $9 \%$ of misorientation angle at $60^{\circ}$ for the as-received sample, the peak of $60^{\circ}$ misorientation angle for LSPed sample with $3 \mathrm{~J}$ pulse energy is $46 \%$ and that for LSPed sample with $\underline{6 \mathrm{~J} \text { pulse energy is } 40 \% \text {. Furthermore, a number of deformation twins of } \sim 60^{\circ}<111>\text { were found in }}$ the surface layer of AISI 304SS subjected to LSP from the EBSD analysis.

(3) For both LSPed samples, the MT width decreases whereas the spacing between two adjacent MTs increases gradually with increasing the distance away from the top surface. Moreover, with increasing pulse energy, the MT width increases whereas spacing is virtually unchanged. Accompanying the existence of MTs, $\varepsilon$-martensitic transformation also occurs during the plastic deformation induced by laser shock wave.

(4) A novel MT-MT intersection with four directions is found for the first time in the plastic 
deformation layer induced by massive LSP treatment, and MT-MT intersection dominates during plastic deformation. On the basis of the impact time at micro-region and the inherited crystal structure of austenite stainless steel, we present an understanding on the maximum of twin planes in FCC alloys with a low SFE. Furthermore, a new grain refinement mechanism based on MT-MT intersection with four directions is developed under the mechanical effect of laser shock wave.

\section{Acknowledgements}

The authors are grateful for the support provided by National Natural Science Foundation of China (No. 51575242), Jiangsu Provincial Natural Science Foundation for Distinguished Young Scientists (No. BK20140012/BE2016148/BK20151341), Major programs of Natural Science Foundation of the Jiangsu Higher Education Institutions (No. 14KJA460002), National Science Foundation for Post-doctoral Scientists of China (No. 2015T80504), and Six Major Talent Peak of Jiangsu Province (2014-ZBZZ-015).

\section{References}

[1] H. Wang, G. Song, G. Tang, Effect of electropulsing on surface mechanical properties and microstructure of AISI304 stainless steel during ultrasonic surface rolling process, Mater. Sci. Eng. A 662 (2016) 456-467.

[2] S. Bagherifard, S. Slawik, I. Fernández-Pariente, C. Pauly, F. Mücklich, M. Guaglianoa, Nanoscale surface modification of AISI 316L stainless steel by severe shot peening, Mater. Des. 102 (2016) 68-77.

[3] C. Correa, L. Ruiz de Lara, M. Díaz, A. Gil-Santos, J.A. Porro, J.L. Ocaña, Effect of advancing direction on fatigue life of 316L stainless steel specimens treated by double-sided laser shock peening, Int. J. Fatigue 79 (2015) 1-9.

[4] H. Lim, P. Kim, H. Jeong, S. Jeong, Enhancement of abrasion and corrosion resistance of duplex 
stainless steel by laser shock peening, J. Mater. Process. Technol. 212 (2012) 1347-1354.

[5] M.P. Sealy, Y.B. Guo, R.C. Caslaru, J. Sharkins, D. Feldman, Fatigue performance of biodegradable magnesium-calcium alloy processed by laser shock peening for orthopedic implants, Int. J. Fatigue 82 (2016) 428-436.

[6] K.Y. Luo, C.Y. Wang, Y.M. Lia, M. Luo, S. Huang, X.J. Hua, J.Z. Lua, Effects of laser shock peening and groove spacing on the wear behavior of non-smooth surface fabricated by laser surface texturing, Appl. Surf. Sci. 313 (2014) 600-606.

[7]I. Nikitin, I. Altenberger, Comparison of the fatigue behavior and residual stress stability of laser-shock peened and deep rolled austenitic stainless steel AISI 304 in the temperature range 25-600 ${ }^{\circ} \mathrm{C}$, Mater Sci Eng A 465 (2007) 176-182.

[8] K.Y. Luo, J.Z. Lu, Y.K. Zhang, J.Z. Zhou, L.F. Zhang, F.Z. Dai, L. Zhang, J.W. Zhong, C.Y. Cui, Effects of laser shock processing on mechanical properties and micro-structure of ANSI 304 austenitic stainless steel, Mater Sci Eng A 528 (2011) 4783-4788.

[9] B.N. Mordyuk, Y.V. Milman, M.O. Iefimov, G.I. Prokopenko, V.V. Silberschmidt, M.I. Danylenko, A.V. Kotko, Characterization of ultrasonically peened and laser-shock peened surface layers of AISI 321 stainless steel, Surf. Coat. Technol. 202 (2008) 4875-4883.

[10] B.S. Yilbas, S.Z. Shuja, A. Arif, M.A. Gondal, Laser shock processing of steel, J. Mater. Process. Technol. 135 (2003) 6-17.

[11] A. Kauffmann, J. Freudenberger, H. Klauß, W. Schillinger, V.S. Sarma, L. Schultz, Efficiency of the refinement by deformation twinning in wire drawn single phase copper alloys, Mater. Sci. Eng. A 624 (2015) 71-78.

[12] K. Wang, N.R. Tao, G. Liu, J. Lu, K. Lu, Plastic strain-induced grain refinement at the nanometer scale in copper, Acta Mater. 54 (2006) 5281-5291.

[13] H.K. Yang, Z.J. Zhang, Z.F. Zhang, Comparison of twinning evolution with work hardening ability in twinning-induced plasticity steel under different strain rates, Mater. Sci. Eng. A 622 (2015) 
$184-188$.

[14] S. Prabhakaran, S. Kalainathan, Compound technology of manufacturing and multiple laser peening on microstructure and fatigue life of dual-phase spring steel, Mater. Sci. Eng. A 674 (2016) 634-645.

[15] S. Prabhakaran, S. Kalainathan, Warm laser shock peening without coating induced phase

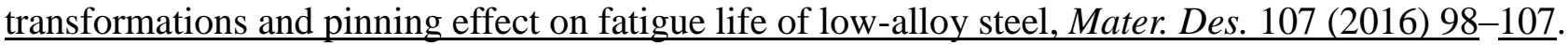

[16] G. Li, S.G. Qu, Y.X. Pan, X.Q. Li, Effects of the different frequencies and loads of ultrasonic surface rolling on surface mechanical properties and fretting wear resistance of HIP Ti-6Al-4V alloy, Appl. Surf. Sci. 389 (2016) 324-334.

[17] P. Tadge, P.K. Gupta, C. Sasikumar, Surface Nano-crystallizationof AISI 304 Stainless Steel through Shot Peening Technique, Mater. Today Proc. 2 (2015) 3245-3250.

[18] Y. Liu, B. Jin, J. Lu, Mechanical properties and thermal stability of nanocrystallized pure aluminum produced by surface mechanical attrition treatment, Mater. Sci. Eng. A 636 (2015) 446-451.

[19] J.Z. Lu, K.Y. Luo, Y.K. Zhang, G.F. Sun, Y.Y. Gua, J.Z. Zhou, X.D. Ren, X.C. Zhang, L.F. Zhang, K.M. Chen, C.Y. Cui, Y.F. Jiang, A.X. Feng, L. Zhang, Grain refinement mechanism of multiple laser shock processing impacts on ANSI 304 stainless steel, Acta Mater. 58 (2010) $5354-5362$.

[20] K.M. Chen, C.C. Zheng, Z.Z. Yuan, J.Z. Lu, X.D. Ren, X.M. Luo, Deformation microstructures of austenitic stainless steel $2 \mathrm{Cr} 13 \mathrm{Mn} 9 \mathrm{Ni} 4$ under ultrafast strain rate by laser shock processing, Mater. Sci. Eng. A 587 (2013) 244-249.

[21] L.C. Zhou, W.F. He, S.H. Luo, C.B. Long, C. Wang, X.F Nie, Laser shock peening induced surface nanocrystallization and martensite transformation in austenitic stainless steel, J. Alloy. Compd. 655 (2016) 66-70.

[22] J.P. Chu, J.M. Rigsbee, G. Banas', H.E. Elsayed-Ali, Laser-shock processing effects on surface 
microstructure and mechanical properties of low carbon steel, Mater. Sci. Eng. A 260 (1999) 260-268.

[23] K.Y. Luo, X. Jinga, J. Shenga, G.F. Sunb, Z. Yan, J.Z. Lu, Characterization and analyses on micro-hardness, residual stress and microstructure in laser cladding coating of 316L stainless steel subjected to massive LSP treatment, J. Alloy. Compd. 673 (2016) 158-169.

[24] K.Y. Luo, H.X. Yao, F.Z. Dai, J.Z. Lu, Surface textural features and its formation process of AISI 304 stainless steel subjected to massive LSP impacts, Opt. Lasers Eng. 55 (2014) 136-142.

[25] G. Kurtuldu, A. Sicco, M. Rappaz. Icosahedral quasicrystal-enhanced nucleation of the fcc phase in liquid gold alloys, Acta Mater. 70 (2014) 240-248.

[26] A.S. Gill, A. Telang, V.K. Vasudevan. Characteristics of surface layers formed on inconel 718 by laser shock peening with and without a protective coating, J. Mater. Process. Technol. 225 (2015) $\underline{463-472 .}$

[27] S.C. Montross, T. Wei, L. Ye, G. Clark, Y.W. Mai, Laser shock processing and its effects on microstructure and properties of metal alloys: a review, Int. J. Fatigue 24 (2002) 1021-1036.

[28] J.Z. Lu, K.Y. Luo, Y.K. Zhang, C.Y. Cui, G.F. Sun, J.Z. Zhou, L. Zhang, J. You, K.M. Chen, J.W. Zhong, Grain refinement of LY2 aluminum alloy induced by ultra-high plastic strain during multiple laser shock processing impacts, Acta Mater. 58 (2010) 3984-3994.

[29] T.S. Byun, On the stress dependence of partial dislocation separation and deformation microstructure in austenitic stainless steels, Acta Mater. 51 (2003) 3063-3071.

[30] L. Xiao, D.L. Chen, M.C. Chaturvedi, Effect of boron on fatigue crack growth behavior in superalloy IN 718 at RT and $650{ }^{\circ} \mathrm{C}$, Mater. Sci. Eng. A 437 (2006) 1-11.

[31] S. Allain, J.-P. Chateau, O. Bouaziz, S. Migot, N. Guelton, Correlations between the calculated stacking fault energy and the plasticity mechanisms in Fe-Mn-C alloys, Mater. Sci. Eng. A 158 (2004) 387-389.

[32] L. Bracke, L. Kestens, J. Penninga, Transformation mechanism of a0-martensite in an austenitic Fe-Mn-C-N alloy, Scripta Mater. 57 (2007) 385-388. 
[33] Y.F. Shen, X.X. Li, X. Sunb, Y.D. Wang, L. Zuo, Twinning and martensite in a 304 austenitic stainless steel, Mater. Sci. Eng. A 552 (2012) 514-522. 


\section{List of Figure Captions}

Fig. 1 Schematic illustration of preparation processes for as-machined sample, the LSPed sample with $3 \mathrm{~J}$ pulse energy, and the LSPed sample with $6 \mathrm{~J}$ pulse energy.

Fig. 2 Three-dimensional and two-dimensional microstructural topographies of the as-received sample and LSPed samples: (a) and (b) 3D profiles at the top surfaces for two LSPed samples, respectively, and (c)-(e) 2D profiles at the top surfaces of as-received sample and two LSPed samples, respectively.

Fig. 3 Typical TEM images at different depths of S2. (a) at the top surface, (b) a magnified image of the box $[\mathrm{M}]$ in (a), (c) corresponding SAED pattern taken from the outlined ellipse $[\mathrm{A}]$ region in (b), (d) at a depth of $30 \mu \mathrm{m}$ from the top surface, (e) a magnified image of the box [N] in (d) and (f) dark-field image.

Fig. 4 Typical TEM images at different depths of S3. (a) at the top surface, (b) a magnified image of the box $[M]$ in (a), (c) corresponding SAED pattern taken from the outlined ellipse [B] region in (b), (d) and (e) at a depth of $30 \mu \mathrm{m}$ from the top surface, and (f) a magnified image of the box [N] in (e).

Fig. 5 EBSD inverse pole figures of AISI 304SS (a) S1, (c) S2 and (e) S3. The color of the grains corresponds to the triangle beside (a) (red: [001], blue: [111], green: [101]). The corresponding histograms of number present versus misorientation angle between neighboring grains are also shown in the below rank: (b) S1; (d) S2 and (f) S3. The peak appearing at $60^{\circ}$ corresponds mostly to twins, since a twin can also be viewed as a rotation of $60^{\circ}$ of the crystallographic lattice around a common [111] direction. (For interpretation of the references to color in this figure, the reader is referred to the web version of the article). 
Fig. 6 Schematic illustration to the formation of the surface roughness and microstructure subjected to massive LSP treatment with different pulse energies. (a) The LSPed sample with $3 \mathrm{~J}$ pulse energy, and (b) the LSPed sample with $6 \mathrm{~J}$ pulse energy. (NOTE: $L$ and $d$ represent the diameter of the effective deformation area and laser spot respectively, and $H E L$ represents Hugoniot Elastic Limit)

Fig. 7 Schematic illustration of microstructural evolution at the top surface of AISI 304SS stainless steel subjected to massive LSP treatment. (a) Dislocation lines in the coarse grain, (b) MT-MT intersections with four directions in the surface layer subjected to massive LSP treatment, and (c) refined grain.

Fig. 8 (a) Three-dimensional of $\{111\}$ twinning planes in the [112]-[110]-[111] ${ }^{[26]}$, (b) a schematic illustration of twin formation during LSP, and (c) $\{111\}$ interplanar angles of $\{111\}$ crystal family. 


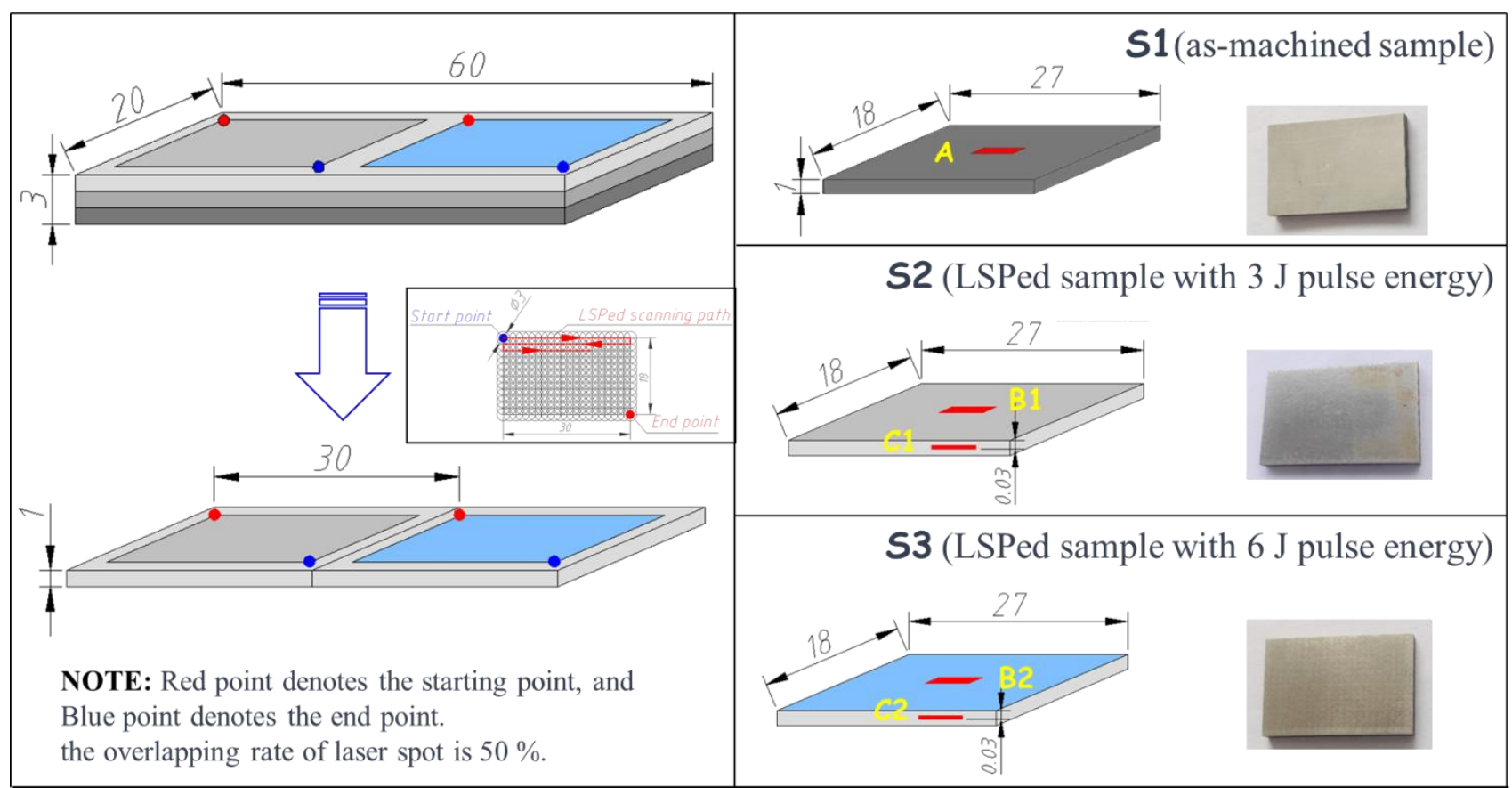

Fig. 1 Schematic illustration of preparation processes for as-machined sample, the LSPed sample with $3 \mathrm{~J}$ pulse energy, and the LSPed sample with $6 \mathrm{~J}$ pulse energy. 

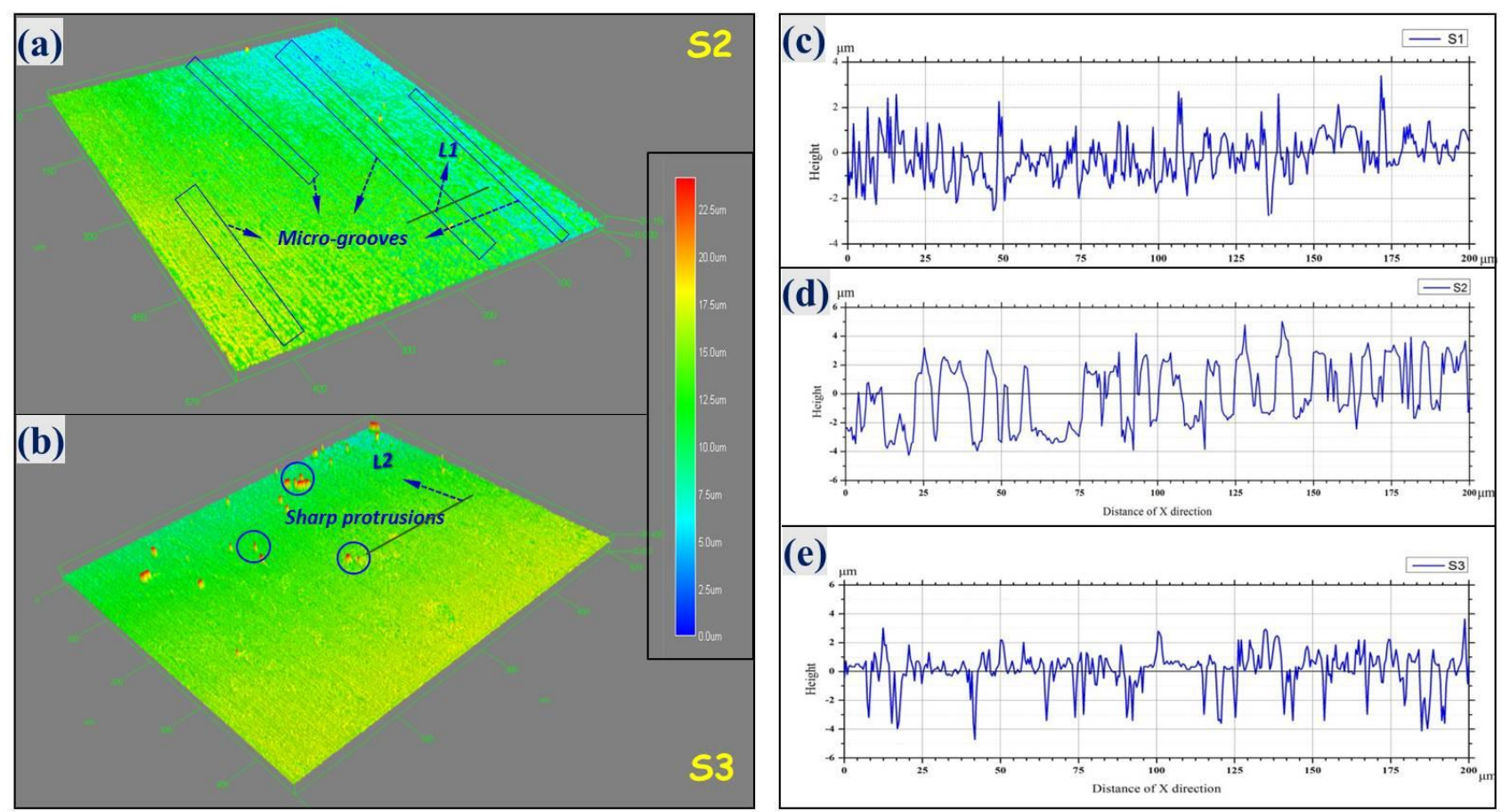

Fig. 2 Three-dimensional and two-dimensional microstructural topographies of the as-received sample and LSPed samples: (a) and (b) 3D profiles at the top surfaces for two LSPed samples, respectively, and (c)-(e) 2D profiles at the top surfaces of as-received sample and two LSPed samples, respectively. 

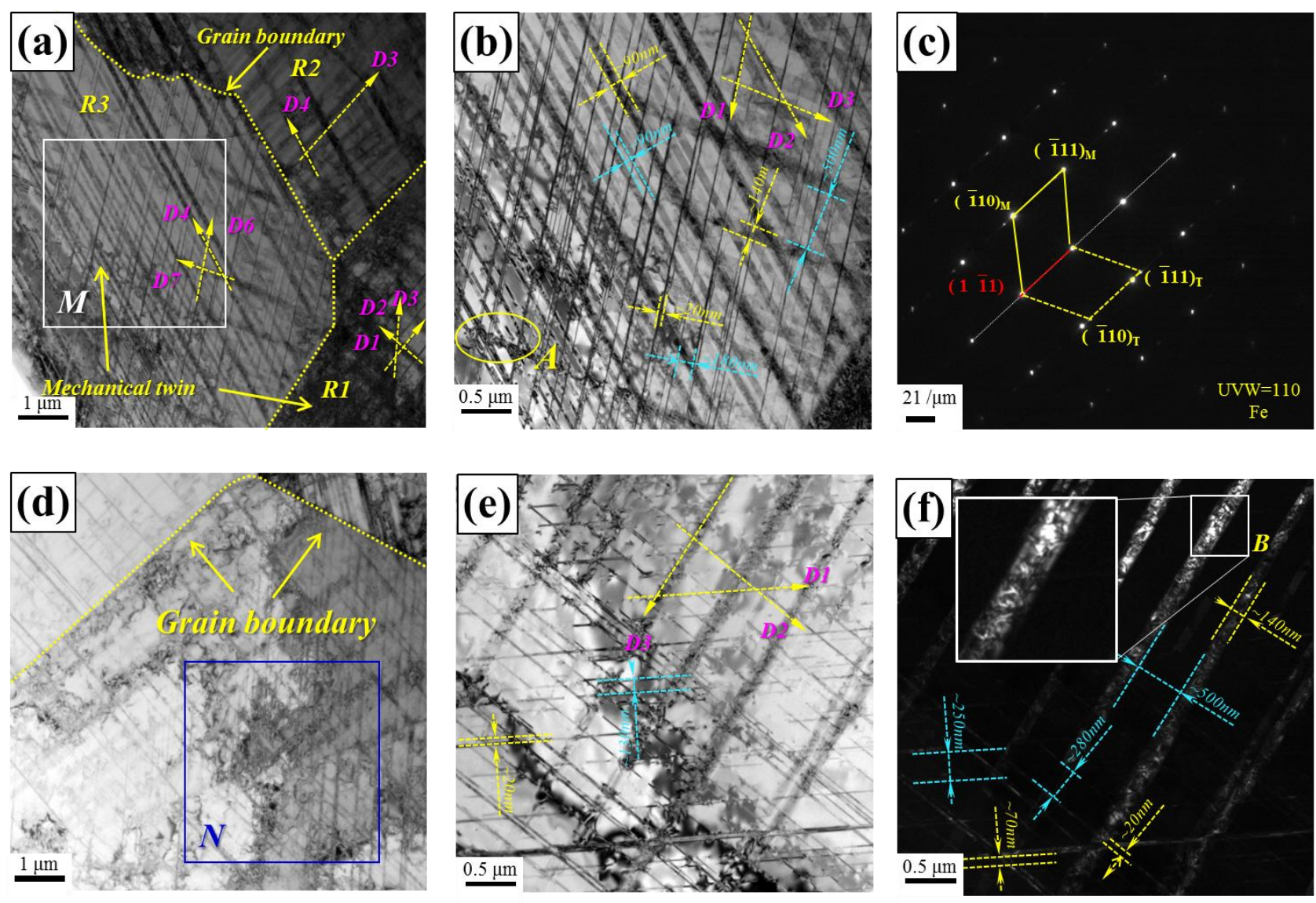

Fig. 3 Typical TEM images at different depths of S2. (a) at the top surface, (b) a magnified image of the box $[\mathrm{M}]$ in (a), (c) corresponding SAED pattern taken from the outlined ellipse [A] region in (b), (d) at a depth of $30 \mu \mathrm{m}$ from the top surface, (e) a magnified image of the box $[\mathrm{N}]$ in (d) and (f) dark-field image. 

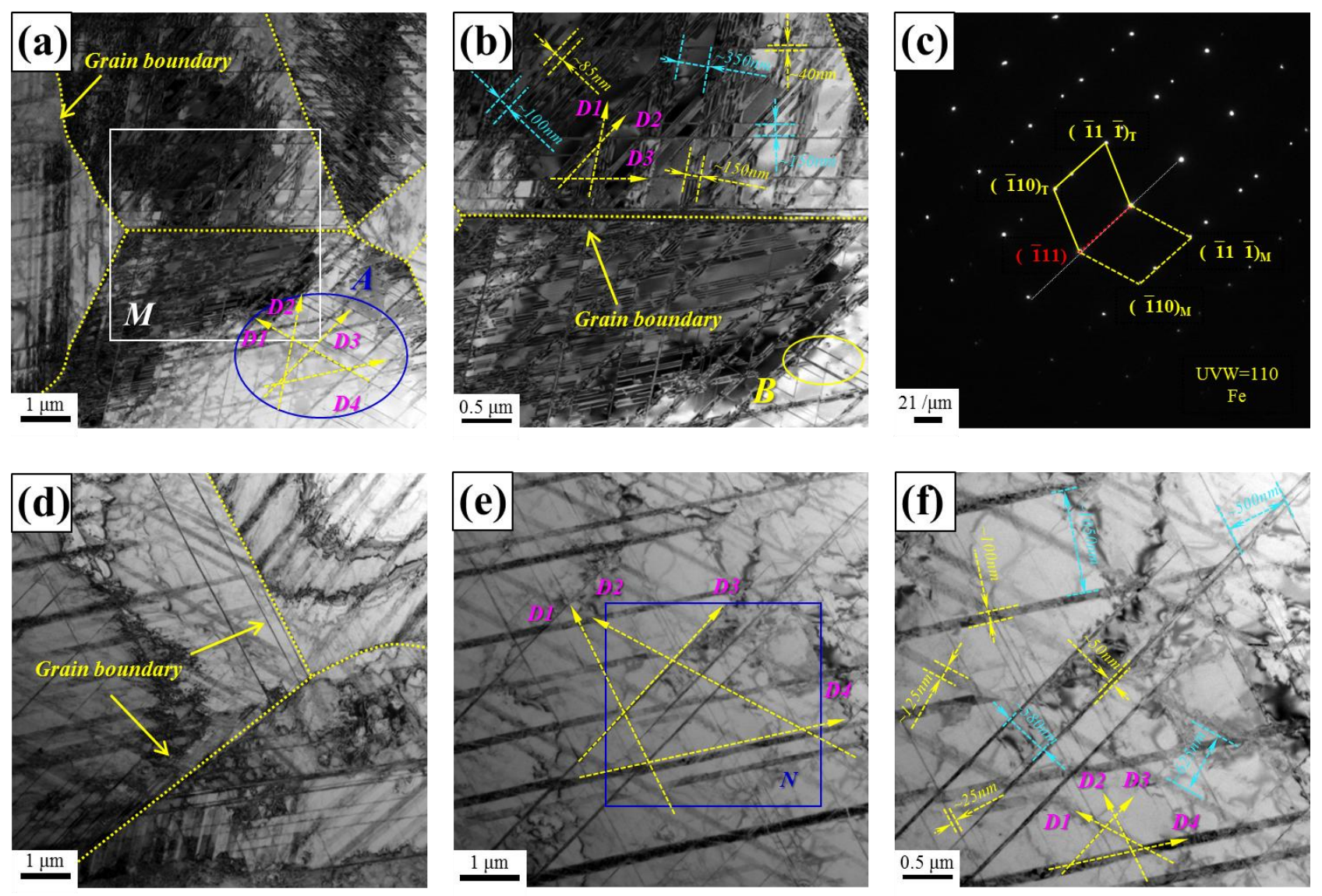

Fig. 4 Typical TEM images at different depths of S3. (a) at the top surface, (b) a magnified image of the box $[\mathrm{M}]$ in (a), (c) corresponding SAED pattern taken from the outlined ellipse [B] region in (b), (d) and (e) at a depth of $30 \mu \mathrm{m}$ from the top surface, and (f) a magnified image of the box [N] in (e). 

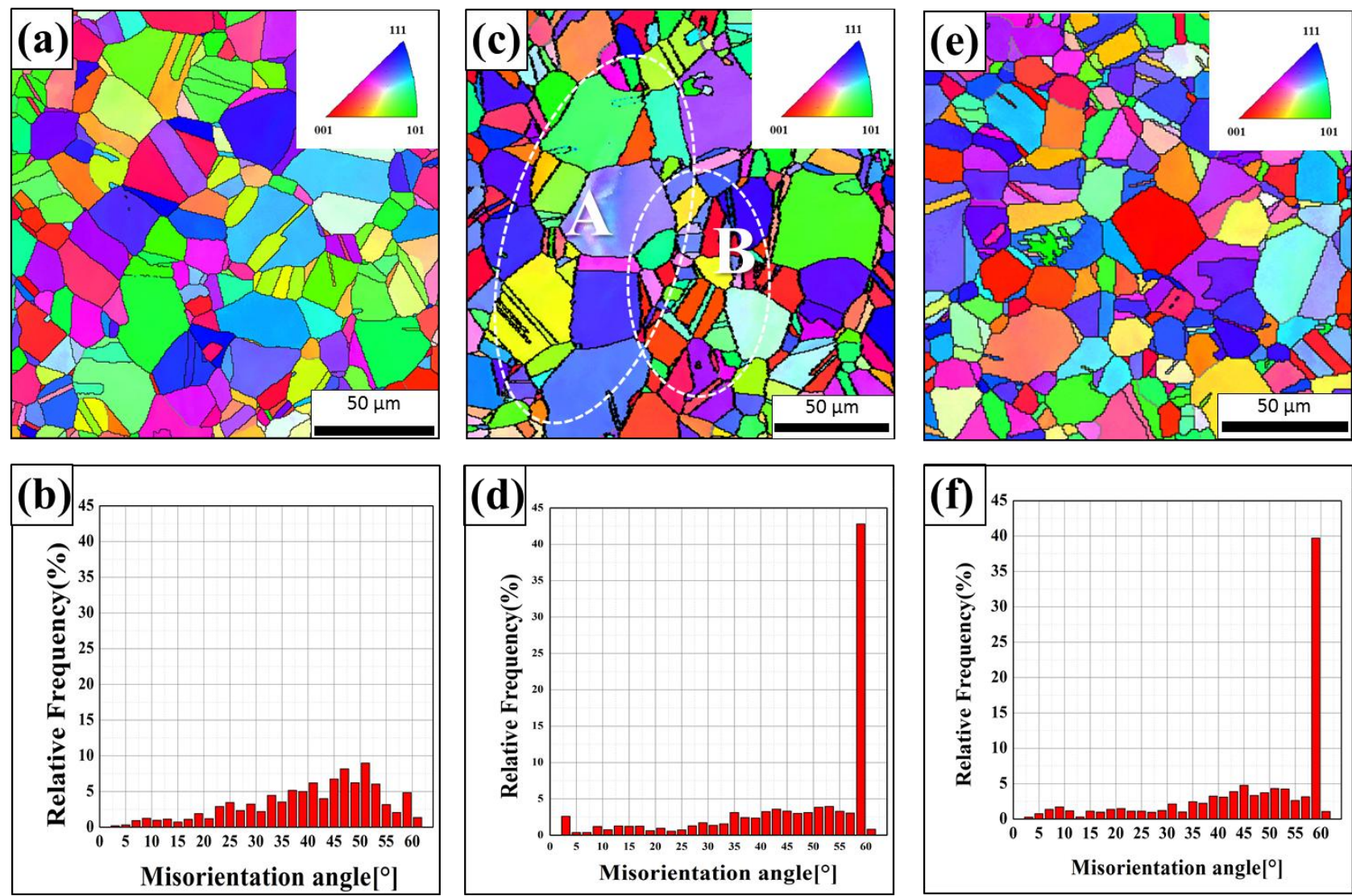

Fig. 5 EBSD inverse pole figures of AISI 304SS (a) S1, (c) S2 and (e) S3. The color of the grains corresponds to the triangle besides (a) (red: [001], blue: [111], green: [101]). The corresponding histograms of number present versus misorientation angle between neighboring grains are also shown in the below rank: (b) S1; (d) S2 and (f) S3. The peak appearing at $60^{\circ}$ corresponds mostly to twins, since a twin can also be viewed as a rotation of $60^{\circ}$ of the crystallographic lattice around a common [111] direction. (For interpretation of the references to color in this figure, the reader is referred to the web version of the article.). 


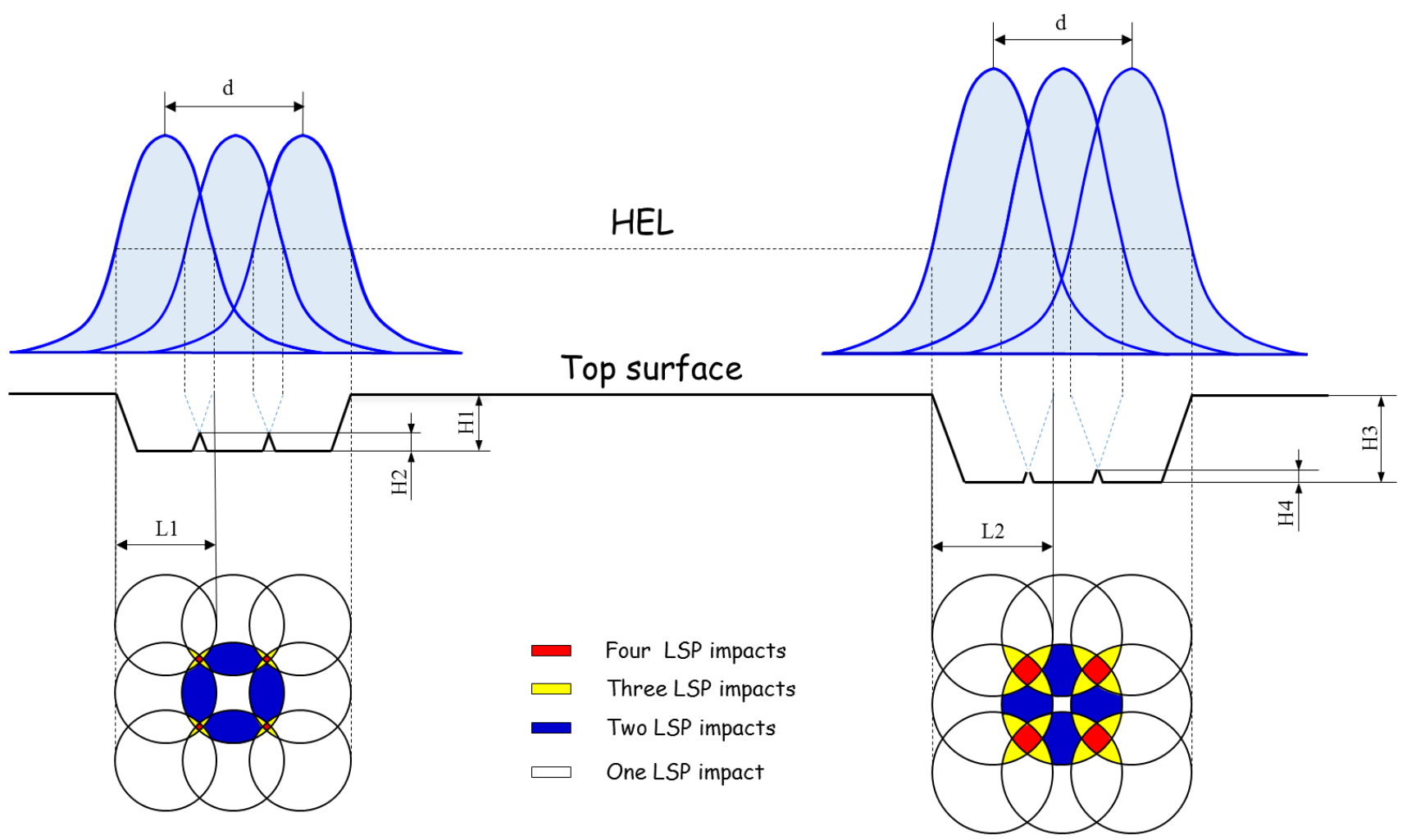

(a) S2

(b) $\mathrm{S3}$

Fig. 6 Schematic illustration to the formation of the surface roughness and microstructure subjected to massive LSP treatment with different pulse energies. (a) The LSPed sample with $3 \mathrm{~J}$ pulse energy, and (b) the LSPed sample with $6 \mathrm{~J}$ pulse energy. (NOTE: $L$ and $d$ represent the diameter of the effective deformation area and laser spot respectively, and $H E L$ represents Hugoniot Elastic Limit) 


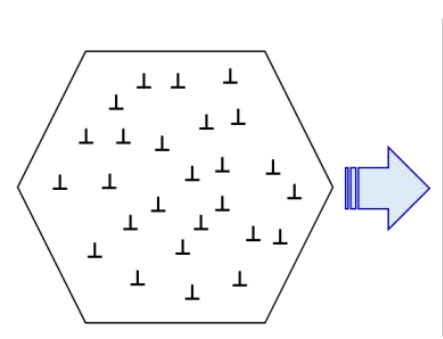

(a)

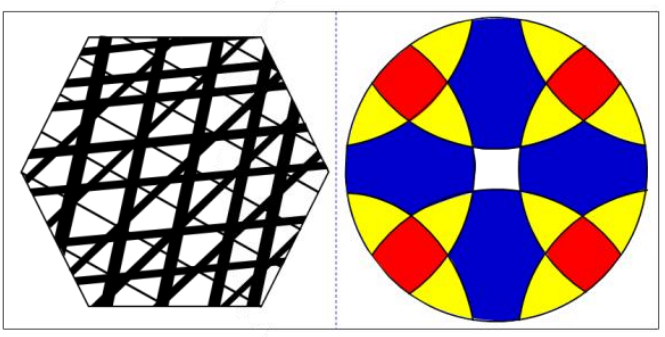

(b)

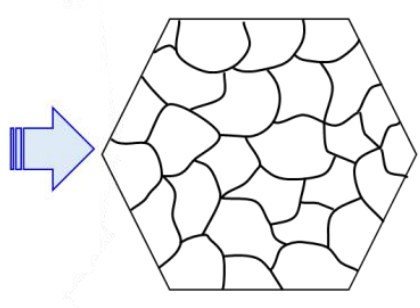

(c)

Fig. 7 Schematic illustration of microstructural evolution at the top surface of AISI 304SS stainless steel subjected to massive LSP treatment. (a) Dislocation lines in the coarse grain, (b) MT-MT intersections with four directions in the surface layer subjected to massive LSP treatment, and (c) refined grain. 
(a)

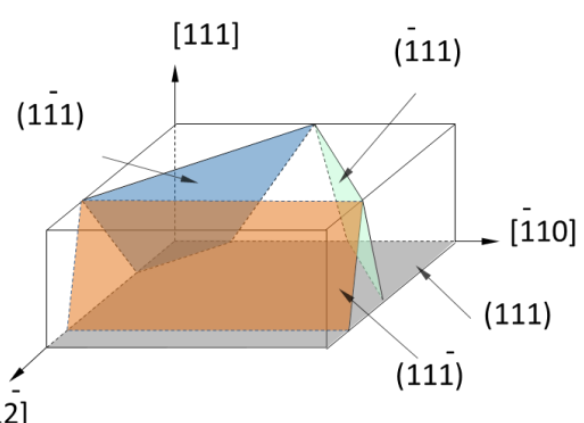

(c)

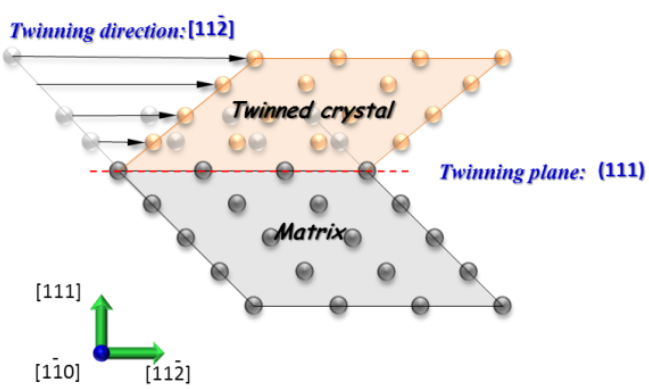

(b)

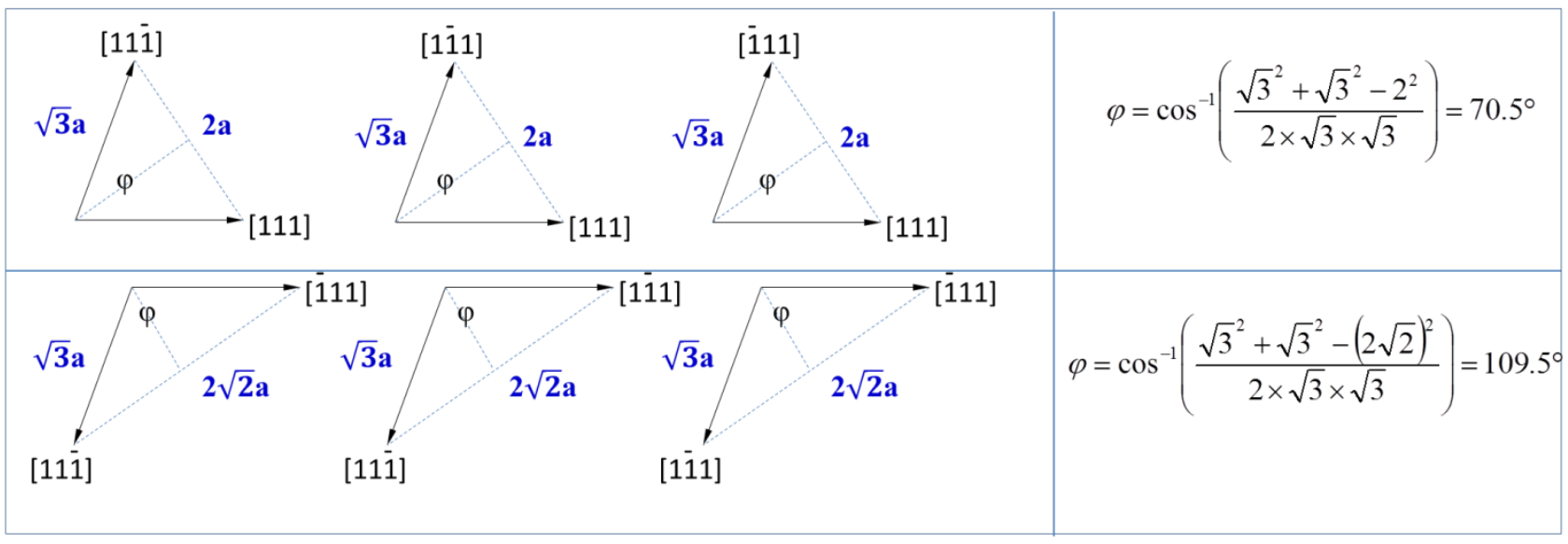

Fig. 8 (a) Three-dimensional of $\{111\}$ twinning planes in the [112]-[110]-[111] ${ }^{[26]}$, (b) a schematic illustration of twin formation during LSP, and (c) $\{111\}$ interplanar angles of $\{111\}$ crystal family. 
Table 1 The chemical composition of AISI 304 stainless steels (wt.\%):

\begin{tabular}{cccccccccc}
\hline Elements & $\mathrm{C}$ & $\mathrm{Mn}$ & $\mathrm{Cr}$ & $\mathrm{M}$ & $\mathrm{Ni}$ & $\mathrm{Cu}$ & $\mathrm{Si}$ & $\mathrm{Nb}$ & $\mathrm{Fe}$ \\
\hline Percent (wt\%) & 0.06 & 1.54 & 18.47 & 0.3 & 8.3 & 0.37 & 0.48 & 0.027 & Balance \\
\hline
\end{tabular}

Table 2 The AVG of Ra for the unprocessed and processed samples by LSP $(\mu \mathrm{m})$

\begin{tabular}{cccc}
\hline Sample & S1 & S2 & S3 \\
\hline AVG of $\mathrm{Ra}(\mu \mathrm{m})$ & 0.537 & 1.226 & 0.947 \\
\hline
\end{tabular}

Table 3 Twinning system of AISI 304 stainless steel

\begin{tabular}{cc}
\hline Twinning plane & Twinning direction \\
\hline$(111)$ & {$[11 \overline{-}]$} \\
$(111)$ & {$[112]$} \\
$(111)$ & {$[1 \overline{-}]$} \\
$(11 \overline{1})$ & {$[112]$} \\
\hline
\end{tabular}




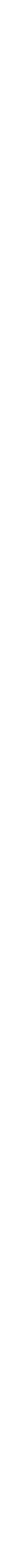

